

\section{DISCLAIMER}

This report was prepared as an account of work sponsored by an agency of the United States Government. Neither the United States Government nor any agency Thereot, nor any of their employees, makes any warranty, express or implied, or assumes any legal liability or responsibility for the accuracy, completeness, or usefulness of any information, apparatus, product, or process disclosed, or represents that its use would not infringe privately owned rights. Reference herein to any specific commercial product, process, or service by trade name, trademark, manufacturer, or otherwise does not necessarily constitute or imply its endorsement, recommendation, or favoring by the United States Government or any agency thereof. The views and opinions of authors expressed herein do not necessarlly state or reflect those of the United States Government or any agency thereof. 


\section{DISCLAIMER}

Portions of this document may be illegible in electronic image products. Images are produced from the best available original document. 


\title{
MECHANICAL PROPERTIES AND MECHANICAL EQUATION OF STATE OF 1100 ALUMINUM ALIOY IN MONOTONIC LOADING
}

\author{
By \\ F. V. Ellis, G. L. Wire and Che $-Y_{\square} \mathrm{L} i$ \\ Department of Materials Science and Engineering \\ Corneli University \\ I thaca, New York 14850
}

\begin{abstract}
Load relaxation experiments are performed on 1100 aluminum alloy as a function of temperature. Based on the experimental results, the behavior of the mechanical equation of state for plastic deformation including the contribution of grain boundary sidding is established. Attempts are made to apply the approach of mechanical equation of state to predict the creep properties of this alloy.
\end{abstract}




\section{INTRODUCTION}

The purpose of this work is to examine the mechanical properties of a commercial alloy at elevated temperatures using the approach of the mechanical equation of state and to test its applicabilities. The properties of interest include strain rate sensitivity, work hardening, grain boundary sliding and creep. It is well known that plastic deformation of a polycrystalline solid is history or path dependent. The concept of mechanical equation of state proposed by Hart ${ }^{(1,2)}$ offers the possibility of using parameters which are path independent to describe plastic deformation. In Hart's approach, an arbitrary deformation path is represented incrementally by

$$
\gamma \mathrm{d} \varepsilon_{p}=\mathrm{d} \ln \sigma-v\left(\mathrm{~d} \ln \dot{\varepsilon}_{p}-Q \mathrm{~d} \frac{1}{\mathrm{RT}^{T}}\right)
$$

where $\gamma=\left.\frac{\partial \ln \sigma}{\partial \varepsilon_{p}}\right|_{\dot{\varepsilon}_{p}}$, and is a work hardening parameter, $\nu=\left.\frac{\partial \ln \sigma}{\partial \ln \frac{\varepsilon_{p}}{p}}\right|_{\varepsilon_{p}}, T$ and is a strain rate sensitivity parameter, $Q=\left.\frac{\partial \ln \dot{\varepsilon}_{p}}{\partial 1 / R T}\right|_{\varepsilon_{p}, \sigma}$ and is an activation energy and $\varepsilon_{p}, \sigma, \dot{\varepsilon}_{p}$ and $T$ are plastic strain, stress, plastic strain rate and temperature respectively. Recent experiments ${ }^{(3-8)}$ have shown the existence of mechanical equation of state for plastic deformation and that both the parameters $\gamma$ and $\nu$ depend only on the current values of stress and plastic strain rate even in the case where grain boundary sliding is important. We can, therefore, integrate Equation 1 using these deformation history independent parameters to describe a deformation path of interest at a given temperature, 
for example creep. It is believed that the present approach based on the concept of mechanical equation of state will offer useful methods for data correlation and extrapolation and savings in mechanical testing. The work reported so far (3-8) has not been carried out to the extent that the integration of Equation 1 is performed, In the present work this step will be accomplished to demonstrate the utility of the approach of mechanical equation of state.

The 1100 aluminum alloy chosen has the advantage that some of its mechanical properties have been reported in the literature ${ }^{(9)}$. In this work load relaxation experiments are performed. The experimental results yield both the deformation behavior of grain matrix and grain boundary and their temperature dependence. The load relaxation data are presented in the form of constant hardness $\log _{10^{\circ}}-\log _{10} \dot{\varepsilon}_{p}$ curves from which the rate sensitivity parameter $v$ can be evaluated. The work hardening parameter of $\mathbf{1 1 0 0}$ aluminum alloy has been measured in a previous work ${ }^{(8)}$. Calculations are made using these two para* meters and Equation 1 to predict the creep behavior of this material.

\section{EXPERIMENTS}

The principle and the detailed experimental procedure of the load relaxation experiment are given in references 3,4 and 10. For experiments at elevated temperatures a tube furnace is used which is placed in a well insulated box. The load cell and the pull-rods are cooled by water from a constant temperature bath. The temperature is controlled by a Leeds \& Northrop 
E1ectromax IV current adjusting type controller. The temperature of the specimen is maintained to within a $1 / 10^{\circ} \mathrm{C}$ fluctuation.

During the load relaxation experiment the specimen is first deformed to the desired plastic strain level. The crosshead is then held fixed. The load relaxation results from converting the elastic strain of the testing system to the plastic strain of the specimen. The load vs time data yield constant hardness $\log _{10} \sigma-\log _{10} \dot{\varepsilon}_{p}$ curves. The overall accuracy of the data is estimated to be five percent. For initial loading, the cross head speed is $.02 \mathrm{in} / \mathrm{min}$ at room temperatur' and $.05 \mathrm{in} / \mathrm{min}$ at hìgher temperature.

The 1100 aluminum a11oy is commercial grade obtained from Peter A. Frasse $\&$ Co. It is solutionized at $550^{\circ} \mathrm{C}$, cold swaged from $3 / 8^{\prime \prime}$ diameter to $0.2^{\prime \prime}$ diameter, tensile specimens machined and annealed at $370^{\circ} \mathrm{C}$ for three days. The specimen has a gage length of 1.5 inch with a diameter of 0.17 inch. The grain size is $100 \mu$.

\section{RESULTS AND DISCUSSION}

1. Load Relaxation Data

Typical load relaxation data obtained at various plastic strain levels and three different temperatures $\left(22^{\circ}, 150^{\circ}\right.$ and $200^{\circ} \mathrm{C}$ ) are shown in Figures $1 \mathrm{a}, \mathrm{b}$ and $\mathrm{c}$ in the form of constant hardness $\log _{10^{\sigma}}-\log _{10} \dot{\varepsilon}_{p}$ curves. These curves are similar to those of lead ${ }^{(7)}$ and high purity aluminum ${ }^{(3)}$ which are also obtained at high homologous temperatures, in that their slope 
increase with decreasing plastic strain rate. A higher slope in these curves corresponds to a smaller stress dependence of plastic strain rate. The present work shows a higher stress dependence based on constant hardness data as compared to that of creap data ${ }^{(9)}$.

The existence of mechanical equation of state requires that these constant hardness $\log _{10} \sigma \cdot \log _{10} \dot{\varepsilon}_{p}$ curves belong to a one parameter family of curves with the arbitrary patameter being the hardness parameter (1). This possibility can be demonstrated if these curves can be translated along a path $\left(\Delta \log _{10} \sigma\right.$ and $\log _{10} \dot{\varepsilon}_{p}$ ) to overlap each other. Recent1y Hart (11) has shown that the constant hardness $\log _{10^{\circ}}-\log _{10} \dot{\varepsilon}_{p}$ curves of high purity aluminum in the region where grain matrix deformation is controlling, can be represented by

$$
\ln \left(\frac{*}{\sigma}-\stackrel{*}{\sigma}^{*}\right)=\left(\frac{\dot{\varepsilon}_{p}}{\varepsilon_{p}}\right)^{\lambda}
$$

where $\lambda$ is a constant which determines the shape of the curve and is independent of temperature, $\stackrel{*}{\sigma}$ is another constant which can be used to measure the hardness of the material, $\dot{\varepsilon}_{p}$ is also a constant and its temperature dependence measures the activation energy $Q$ and $\stackrel{*}{\sigma}$ and $\stackrel{*}{\varepsilon}_{p}$ are related through the translation path. The constant hardness $\log _{10} \sigma-\log _{10} \dot{\varepsilon}_{p}$ curves for each temperature in Figures $1 a, b, c$ are found to belong to a one parameter family of curves. The translation path has a stope $\left(\left.\frac{d \ln \sigma}{d \ln \varepsilon_{p}}\right|_{v}\right)$ of approximately 0.2. For each temperature the curves are found to fit Equation 2 well with a constant $\lambda$. But the value of the parameter $\lambda$ is found to vary with temperature. 
At elevated temporatures grain boundary sliding will contribute to plastic deformation ${ }^{(7)}$. The co-existence of grain boundary sliding and grain matrix deformation has been shown to alter the shape of $\log _{10} \sigma-\log _{10} \bar{\varepsilon}_{p}$ curves (7). It is therefore not unreasonable to find in the present work that the value of the parameter $\lambda$ (or the shape of these curves) dependent on temperature. This possibility will be analyzed in the section on grain boundary sliding.

\section{Temperature Dependence of Constant Hardness $10 \mathrm{~g}_{10} 0$}

$$
\underline{\log }_{10} \dot{\varepsilon}_{p} \text { Curves }
$$

The activation energy, $Q$, for plastic deformation is an important parameter if temperature is to be included as a variable in the consideration of mechanical equation of state. In principle the activation energy $Q$ can depend on stress, plastic strain rate and temperature. It will be convenient if the activation energy appearing in Equation 1 depends only on temperature and not on stress and plastic strain rate. This possibility can be tested only be experiment. For the case where the activation energy $Q$ depends on $1 y$ on temperature and the shear modulus is temperature independent we should be able to translate the $\log _{10} \sigma \log _{10} \dot{\varepsilon}_{p}$ curves of two temperatures but of the same hardness along the $\log _{10} \dot{\varepsilon}_{p}$ axis to overlap each other. The activation energy $Q$ can be evaluated by $\Delta \ln \dot{\varepsilon}_{p}=Q \Delta\left(\frac{1}{R T}\right)$. It should be noted as discussed above that the $\log _{10^{\circ}}-\log _{10} \dot{\mathrm{E}}_{\mathrm{p}}$ curves at elevated temperature will include the influence of grain boundary siding $(7)$. The activation energy for grain boundary sliding may be different from that for grain matrix deformation. 
Caution should be exercised in the interpretation of the experimental data.

Experimentally in order to obtain constant hardness $\log _{10^{\circ}}$ $\log _{10} \dot{E}_{p}$ data on one specimen over a wide temperature range we take advantage of the results ${ }^{(8)}$ that at high hardness $\left(\log _{10}{ }^{*}\right)$. and low stress the work hardening parameter $\Gamma\left(\left.\frac{\partial \ln \sigma^{*}}{\partial \varepsilon_{p}}\right|_{\varepsilon_{p}}\right)$ for 1200 aluminum alloy is a small number. We begin the load relaxation experiments at room temperature using a heavily cold worked specimen $\left(\right.$ high $\left.\log _{1} 0^{*}\right)$. After the room temperature test, we rajse the temperature of the system and reload the specimen to the desired stress level for the load relaxation experiment at a higher temperature. The reloading is carried out in such a way that sufficient deformation is introduced to minimize the transient effects during load relaxation $(3,7)$ but without increasing the hardness of the specimen. At higher temperatures the stresses involved are lower compared to the room temperature values. Since we have a specimen with high hardness $\left(\log _{10^{\circ}}\right)$, it will be difficult at the lower stresses to harden the specimen further with moderate plastic strain increment during reloading and therefore ensure the hardness of the specimen to remain the same. The absence of recovery effects in the temperature range of this work will be discussed in a later section.

Typical constant hardness $\log _{10} \sigma-\log _{10} \dot{\varepsilon}_{p}$ curves at various temperatures obtained using the above approach are shown in Figure 2. It is seen from the figure that as the temperature increases, the stress decreases and the slope of the $\log _{10} \sigma \cdot \log _{10} \dot{\epsilon}_{p}$ curves increases. The shape of these curves cannot be represented by the same value of the parameter $\lambda$ using Equation 2 suggesting 
as pointed out previously the possibility of the existence of grain boundary sliding.

It should be noted that the curves in Figure 2 are plotted on a normalized $\log _{10} \frac{\sigma}{G}$ where $G$ is shear modulus. The temperature dependence of the modulus is calculated using the data and method given in references 13 and 14 . The stress scale shown is for roon temperature experimental values. The amount of correction in $\Delta \log _{10^{\circ}}$ is $0.029,0.043,0.050,0.057$ and 0.064 between room temperature and $125^{\circ} \mathrm{C}, 175^{\circ} \mathrm{C}, 200^{\circ} \mathrm{C}, 225^{\circ} \mathrm{C}$ and $250^{\circ} \mathrm{C}$ respectively.

In the absence of grain boundary sliding Hart's suggests that the high stress portion of the high temperature $\log _{10}$ o $\log _{10} \dot{\varepsilon}_{p}$ curves can be translated along the $\log _{10} \dot{\varepsilon}_{p}$ axis to overIap the low stress portion of the Iow temperature curve according to Equation ${ }^{(12)}$. Similar behavior is found in the present data even though the existence of grain boundary sliding is suggested. In Figure 3 , the curves in Figuxe 2 are translated along the $\log _{10} \dot{\varepsilon}_{p}$ axis to overlap each other and form a continuous curve. During this exercise the room temperature curve is fixed and the $250^{\circ} \mathrm{C}$ is shifted with the largest $\Delta \log _{10^{\circ}} \dot{\varepsilon}_{p}$ with the lower temperature curves translating progressively less. The significance of the continuous composite curve will be discussed in the next section. From figure 3 it is seen that the measured curves can be translated to overlap each other well except for the initial portion of the curve due to transient effect $(3,7)$. These results suggest that the activation energy $Q$ in Equation 1 is independent of stress and plastic strain rate. From the $\Delta \log _{10} \dot{\varepsilon}_{p}$ value between two neighboring temperatures we can evaluate 
corresponding $Q^{\prime} s$. We find the activation energy is $33.5 \mathrm{Kcal} /$ mole, $34.6 \mathrm{Kcal} / \mathrm{mole}, 27.7 \mathrm{Kcal} / \mathrm{mole}, 28.3 \mathrm{Kcal} / \mathrm{mole}$ and 20.4 Kcal $/$ mole between $250^{\circ} \mathrm{C}$ and $225^{\circ} \mathrm{C}, 225^{\circ} \mathrm{C}$ and $200^{\circ} \mathrm{C}, 200^{\circ} \mathrm{C}$ and $175^{\circ} \mathrm{C}, 175^{\circ} \mathrm{C}$ and $125^{\circ} \mathrm{C}$, and $125^{\circ} \mathrm{C}$ and room temperature respectively. It should be noted that the values of activation energy given here are different from those obtained using Equation 1 where modulus correction is not included. These calculated values of activation energy are in agreement with those found by creep experiments $(9,15)$. In the creep experiments it is found also that the activation energy $Q$ decreases with decreasing temperature. The significance of the calculated activation energy will be discussed also in the next section. We can conclude however that the temperature dependence of constant hardness $\log _{10^{\sigma}}$ $\log _{10} \dot{\varepsilon}_{p}$ curves of 1100 aluminum alloy can be correlated with an activation energy which is temperature dependent and is independent of stress and plastic strain rate.

\section{Grain Boundary S1iding}

In the discussion of grain boundary sliding, the concept introduced by Hart $(16,17)$ will be adopted. According to Hart at high stresses and/or low temperatures the deformation of a polycrystalline solid, will be controlled by grain matrix processes. At intermediate stresses and/or temperatures the contribution of grain boundary sliding will becone important. In this region the deformation will be controlled by grain matrix and by grain boundary sliding with plastic compatibility being the constraint. As stress decreases and/or temperature increases further, grain boundary will offer negligible resistance. The deformation of 
a polycrystalline solid will be again controlled by grain matrix processes with essentially relaxed or free sliding grain boundary. The load relaxation data in the form of a constant hardness $\log _{10^{\sigma}}-\log _{10} \varepsilon_{p}$ curve, will show therefore grain matrix behavior in the portion of high plastic strain rate. As plastic strain rate decreases, with increasing contribution of grain boundary sliding the $\log _{10} \sigma-\log _{10} \dot{\varepsilon}_{p}$ data will deviate from grain natrix curve and show a higher increase in the slope of the $\log _{10} \sigma-\log _{10} \dot{e}_{p}$ curve as compared to that for grain matrix curve. At very low plastic strain rates the data will exhibit grain matrix behavior again. In the last region where the grain boundary is relaxed the data will fall on a grain matrix curve which is translated by a finite $\Delta \log _{10} \sigma$ and $\Delta \log _{10} \dot{\varepsilon}_{p}$ from the grain matrix curve for high strain rates where grain boundary is not relaxed. Hart has proposed two phenomenological models to include both the contribution of grain boundary sliding and grain matrix deformation $(16,17)$. In the so-called parallel mode ${ }^{(16)}$, the grain matrix curves with relaxed grain boundary is translated by a finite $\Delta \log _{10} \sigma$ from the grain matrix curve with unrelaxed grain boundary. In the series model the translation is by a finite $\Delta \log _{10} \dot{\varepsilon}_{p}$ on $1 y$. Other possibilities exists which will involve a translation of $\Delta \log _{10} 0^{\circ}$ and $\Delta \log _{10} \dot{\varepsilon}_{p}$ both. At the present there is no theoretical reason to prefer one over the other anong these possibilities. Within our experimental limitation, the present data show that the parallel model seems to be more reasonable compared to the series model, but we cannot rule out a translation invloving both $\Delta \log _{10}$ and $\Delta \log _{10} \dot{E}_{p}$. For simplicity the following disucssion will be based on the parallel model. This choice should be taken as an assumption. 
Following Hart ${ }^{(16)}$ the parallel model can be represented by

$$
\begin{aligned}
& \sigma=Y \sigma_{F}+(1-Y) \sigma_{S} \\
& \dot{\varepsilon}=\dot{\epsilon}_{S}+\dot{\varepsilon}_{g} \\
& \ln \left(\stackrel{*}{\sigma} / \sigma_{F}\right)=\left(\frac{\stackrel{*}{E}}{\dot{E}}\right)^{\lambda} \\
& \ln \left(\stackrel{*}{\sigma} / \sigma_{S}\right)=\left(\frac{\stackrel{*}{c}}{\dot{\epsilon}_{\mathrm{S}}}\right)^{\lambda} \\
& \sigma_{S}=K_{g} \dot{\varepsilon}_{g}^{\mu}
\end{aligned}
$$

where subscripts $F$ and $S$ denote two parallel elements; the $S$ element contains an element with grain matrix behavior represented ${ }^{-}$ by Equation $3 d$ and a grain boundary element represented by Equation 3 with subscript $g$, these two elements are in series; the F element is represented by Equation $3 \mathrm{c}$; the parameter $Y$ measures the $\Delta \log _{10} \sigma$ translation required to produce the grain matrix $\log _{10} \sigma-\log _{10} \dot{\epsilon}_{p}$ curve with relaxed grain boundary. According to this model at high plastic strain rates, grain boundary is unrelaxed, $\dot{\varepsilon}_{g} \ll \dot{\varepsilon}_{S}$ and $\sigma_{F}=\sigma_{S}=\sigma$ resulting in pure grain matrix behavior. At low plastic strain rates, the grain boundary is completely relaxed, $\sigma_{S} \rightarrow 0$ and grain matrix behavior will be controlling again.

The data shown in Figure 3 will be analyzed to show that they exhibit the entire range of behaviors discussed above. At high stresses and/or low temperatures grain matrix process predominates which is represented by the upper solid curve. At low stresses and/or high temperatures the data fall on the lower dashed line representing grain matrix behavior with relaxed grain boundary. In the intermediate stresses and/or temperatures both 
the contributions of grain boundary sliding and grain matrix deformation are important.

A unique feature of the data shown in Figure 3 as discussed previously is that the constant hardness $\log _{10} \sigma-\log _{10} \dot{\varepsilon}_{p}$ curves of different temperatures can be translated along the $\log _{10} \dot{\varepsilon}_{p}$ axis to overlap each other and form a continuous curve. Direct evidence for the existence of grain boundary sliding in 1100 aluminum alloy in the present temperature range can be found in the Iiterature ${ }^{(9)}$. The data in Fig. 3 suggests therefore that with the presence of grain boundary sliding the constant hardness $\log _{10} \sigma-\log _{10} \dot{\varepsilon}_{p}$ curves of different temperatures still belong to a single temperature compensated curve. This is possible according to the model described by Equation 3 only if (1) the con-

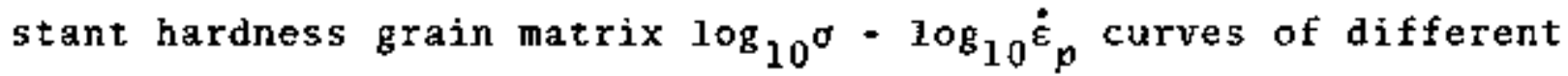
temperatures have the same shape (same value of $\lambda$ in Equations $3 c$ and $3 d$ ), (2) the grain boundary $\log _{10} \sigma-\log _{10} \dot{\varepsilon}_{p}$ curves (Equation $3 e$ ) for different temperatures but belong to a material of constant hardness also have same shape, and (3) the activation energy $Q$ for grain boundary sliding equals to that for grain matrix deformation. It should be noted that if these requirements are satisfied, a polycrystalline solid will exhbit the behavior shown in Figure 3 independent of the choice of deformation model. The $\log _{10} \sigma$ and $\log _{10} \dot{E}_{p}$ scales shown in Figure 3 are for room temperature. If the above requirements are satisfied for a higher temperature of interest the $\log _{10} \sigma$ value can be obtained by correcting for modulus effects described previously and the $\log _{10} \dot{\varepsilon}_{p}$ value can be obtained by correcting for $\Delta \log _{10} \dot{\varepsilon}_{p}=f \frac{0}{2.3} \mathrm{~d}\left(\frac{1}{R T}\right)$. At the present we sha11 take the three requirements 1 isted above 
as assumptions and proceed with the analysis.

In the analysis the grain matrix curve (Equation 2) is obtained from the portion of the $250^{\circ} \mathrm{C}$ data shown in Figures 2 and 3 which exhibit grain matrix behavior. The parameter $\lambda$ is found to be 0.11 . The $\log *^{*}$ value and the $\log _{10} \stackrel{*}{\dot{\varepsilon}}$ value are found to be 4.125 and -14.5 respectively by matching the grain matrix curve on to the room temperature curve. The $\Delta \log _{10} \sigma$ value between the upper and lower matrix curves in Figure 3 is 0.1 corresponding to a $Y$ value of 0.8 . With these two matrix curves defined we can use experimental $\log _{10} \circ$ and $\log _{10} \dot{\varepsilon}_{p}$ values and equations $3 \mathrm{a}, 3 \mathrm{~b}, 3 \mathrm{c}$ and $3 \mathrm{~d}$ to solve for $\sigma_{\mathrm{F}}, \sigma_{\mathrm{S}}, \dot{\varepsilon}_{\mathrm{S}}$ and $\dot{\varepsilon}_{\mathrm{g}}$. The resulting grain boundary curves for different temperatures in the form of a $\log _{10}{ }^{\sigma} \mathrm{s} v \log _{10} \dot{\varepsilon}_{\mathrm{g}}$ plot are shown in Figure 4 . In Figure $\mathrm{s}$ these grain boundary curves are translated along the $10 g_{10} \dot{\varepsilon}_{\mathrm{g}}$ axis using the activation energy $Q$ determined in the previous section to form a continuous curve. It is seen from Figures 4 and 5 that considerable scatter of the calculated values exist. The scatter results mainly from computation where differences between two nearly equal numbers are taken. The initial portion (high strain rates region) of the grain boundary curve does not fit on to the composite curve in Figure 5 well. This portion of the grain boundary curve corresponds to the portion of the data shown in Figure 3 which also do not fit on to the composite curve due to the existence of transient effects $(3,7)$.

It is interesting to note that the composite grain boundary curve shown in Figure 5 exhibits nearly Newtonian viscous behavior at low stresses and/or high temperatures. As the stress increases and/or temperature decreases, the stress dependence of the grain 
boundary sliding rate becomes increasing non-Newtonian. This result is consistent with the results given in references 7,18 and 19 and demonstrates the importance of local accomodation by grain matrix deformation during grain boundary sliding. It is therefore not unreasonable to assume that the activation energy for grain boundary sliding is the same as that for grain matrix deformation. Horton (19) has found similar results in his work. The present analysis suggests therefore that the activation energy Q reported in the previous section is for grain boundary sliaing and grain matrix deformation both.

The present analysis shows that at low plastic strain rates, grain boundary sliding will become important even at room temperature for heavily cold worked material (high $\log _{10^{\circ}}{ }^{*}$ ). This possibility may be supported by the direct observation of grain boundary sliding at room temperature in aluminum $(20)$. The fact that both the grain boundary curves (Figure 4) and the measurea $\log _{10} \sigma-\log _{10} \dot{\varepsilon}_{p}$ curves (Figure 2) can be translated to form a continuous temperature compensated curve, may be used to support the assumptions made for requirements (1) and (2) discussed previously. We note that the exact shape of grain boundary curve is model dependent. Based on the present analysis we may make conclusions however which are model independent. These are (1) grain boundary sliding in 1100 aluminum alloy will show a range of stress dependence depending on the experimental condition and (2) the activation energy for grain boundary sliding is close to that for grain matrix deformation. 


\section{Recovery}

In the discussion so far the recovery of deformed structure in the temperature range of the present work has been assumed to be negligible. The viewpoint taken here is that at temperatures below those where recrystallization and sub-boundary migration are possible, recovery is not important. The temperature range in the present work is below that found experimentally for recrystallization $(9,2 l)$. In our previous work on lead, the accelerated stress drop during stress relaxation is observed and is assocaited with grain boundary migration (?). This phenomenon is not found in the present work. The absence of accelerated stress drop can be used also to show that we do not have recrystallization.

In order to establish experimentally that the data shown in Figure 2 involve only one hardness, after the $250^{\circ} \mathrm{C}$ test we preform load relaxation experiments at room temperature. This procedure is similar to that described in reference 3 to test if the room temperature $\log _{10} \sigma-\log _{10} \dot{\varepsilon}_{p}$ curves before and after the high temperature runs have the same hardness $\left(\log _{10^{\circ}}\right)$. This experiment is not completely successful as the specimen is overstrained during reloading. From the experimental results we can conclude however that if recovery has occurred the change in $\log _{10} \stackrel{\star}{\sigma}$ value is less than 0.02 .

\section{Creep Analysis}

The behavior of mechanical equation of state demonstrated by the present work suggests that the strain rate sensitivity parameter is history independent and can be evaluated by Equation 2. In 
our previous work we have shown that the work hardening parameter $Y$ is also history independent ${ }^{(8)}$. We can therefore integrate Equation 1 using these parameters to describe an arbitrary deformation path of interest. In this section we shall use this approach to calculate the constant load creep behavior of 1100 aluminum alloy and to test the applicability of the present approach .

We shall limit the analysis in the high temperature and low strain rate region where the parameter $v$ can be evaluated from constant hardness curves equivalent to the lower dashed curve shown in Figure 3. The room temperature $\log _{10} \sigma$ value shown in Figure 3 , for example, has to be corrected for modulus effect described previously to obtain the high temperature values. Similarly the strain rate shown in Figure 3 can be converted to corresponding high temperature strain rate using the activation energy reported previously such that $\Delta \log _{10} \dot{\varepsilon}_{p}=f \frac{Q}{2.3} d(1 / R T)$.

The work hardening properties of 1100 aluminum alloy have been reported in reference 8 . We require an analytical expression to correlate these data in terms of o and ${ }_{\sigma}^{\star}(8)$. The values of work hardening parameter $\Gamma$ given in reference 8 are reproduced in Figures 6 in a plot of $\log _{10}{ }^{*}$ vs $\log _{10} \frac{\stackrel{*}{\sigma}}{\sigma}$ where the $\log _{10} \sigma$ and the $\log _{10} \frac{*}{\sigma}$ coordinates correspond to experimental values at room temperature. The parameter $\Gamma$ is $\left.\frac{\partial \ell_{n} \stackrel{*}{a}}{\partial \varepsilon_{p}}\right|_{p}=\frac{\mu}{\mu-\nu} \gamma$. Additional high temperature values of the parameter $F$ are included in Figure 6 . These $\Gamma$ values as a function of $\sigma$ and $\stackrel{*}{\sigma}$ are obtained using the values of $\gamma$ from constant strain rate $\log _{10} \sigma \cdot \varepsilon_{p}$ data $(8)$ and the grain matrix values of $\sigma, \stackrel{*}{\sigma}$ and $v$. Complications arise here for the data analysis because the experimental stress and plastic strain rate of these additional data are either in the 
region where both grain boundary sliding and grain matrix deformation are important or in the region, where the grain boundary is relaxed and we requixe information on grain matrix hardening due to strain. For example the high plastic strain rate region of the $175^{\circ} \mathrm{C}$ data which have been translated to the room temperature coordinates shown in Figure 3 do not fall on the upper grain matrix curve (upper solid line). The plastic strain rates in this region are used in obtaining the work hardening data. In the case where parallel model is applicable, the required grain matrix $\sigma$ and $v$ values can be found at the same plastic strain rate from the corresponding grain matrix curve (upper solid line in Figure 3) which also gives the required $\stackrel{*}{o}$ values. After translation for the temperature effects, if a pair of stress and - plastic strain rate do not, for example, fall on the composite curve in Figure 3, the corresponding grain matrix properties can be found by first translating the curves in Figure 3 along a path with slope taken as $\mu=0.2$, such that the translated composite curve passes through the $\log _{10^{\sigma}}$ and $\log _{10} \dot{\varepsilon}_{p}$ point of interest. The desired $\stackrel{*}{\sigma}$ value is obtained from the translated upper solid curve and the desired $\sigma$ and $v$ values are found at the same $\log _{10} \dot{\bar{\varepsilon}}_{p}$ value a1so from the translated upper solid curve. The exercise described above assumes that (1) the parallel model adopted previousiy is valid, (2) the grain boundary curves (Figure 5) translate similarly to the grain matrix $\log _{10^{\sigma}}-\log _{10} \dot{\varepsilon}_{p}$ curves. The latter assumption can be supported from the results that the higher temperature constant hardness curves from load relaxation experiments which include the grain boundary sliding contribution, translate with a path with a slope of 0.2 within the 
measured range.

We assume for simplicity that the data shown in Figure 6 can be correlated by constant $r$ lines in the form of parallel straight lines. These constant $\Gamma$ lines can be expressed by $\Gamma=A \sigma^{\alpha} / \sigma^{*}$ where $A=1.17 \times 10^{20}, \alpha=7.82$ and $B=12.5 ; \Gamma$ is dimensionless and $\sigma$ and $\stackrel{*}{\sigma}$ are in psi. It is interesting to note that the stress - plastic strain relationship calculated based on the above expression is consistent with the power relationship mentioned in reference 8 .

He can represent the information required for creep calculations in analytical expressions. In addition to Fquations 1 and 2 they are

$$
\begin{gathered}
\nu=\lambda \ln \stackrel{*}{\sigma} / \sigma \\
\Gamma=A \sigma^{\alpha} / \sigma^{\beta}=\frac{\mu}{\mu-v} \gamma \\
\ln \stackrel{*}{\sigma}=\mu \ln \stackrel{*}{\dot{E}}_{p}+\ln \mathrm{D}
\end{gathered}
$$

where Equation 6 representing the translation path is derived from Equation 2 and the values of the constants for creep calculation at $250^{\circ} \mathrm{C}$ are $\lambda=0.11, \dot{A}=0.60 \times 10^{20}, a-7.82, \beta=12.5$, $\mu=0.2$ and $D=10^{6.75}$. We note that the coordinates of the data in Figures 3 and 6 are room temperature experimental values for which $A=1.17 \times 10^{20}$ and $D=10^{7.025}$. The D value used for creep calculation is found by load relaxation experiments at room temperature on creep specimens of slightly different microstructure. It has been corrected for modulus effect to $250^{\circ} \mathrm{C}$ but does not include the translation for thermal activation. The value of the paraneter $A$ at $250^{\circ} \mathrm{C}$ is obtained by correcting for the temperature dependence of shear modulus for both $\sigma$ and $\stackrel{*}{*}$. 
In Figure 7 the data presented in Figures 3 and 6 are extrapolated with the aid of analytical relationships given previously to illustrate the ability of the present approach to cover a wide range of deformation variables for creep calculations. In this figure Equation 2 representing grain matrix behavior is plotted for various values of $\stackrel{*}{\circ}$. The slope of these constant hardines curves gives the value of $\nu$ for creep calculation. In the same figure constant $\Gamma$ lines based on Equation 5 are given. From Equation 5 one may also obtain the $\Gamma$ value for a given $\log _{10} 0^{\circ}$ and $\log _{10}{ }^{\star} \sigma$ pair from the $\log _{10}\left(\Gamma{ }_{\sigma}^{*} B / A\right)^{1 / \alpha}$ coordinate.

From the Appendix the integration of Equation 1 for constant. stress can be accomplished by

$$
\begin{gathered}
\varepsilon_{p}=\frac{1}{\beta A \sigma^{\alpha-\beta}}\left[\exp \left(\frac{\beta \nu}{\lambda}\right)-\exp \left(\frac{\beta v_{0}}{\lambda}\right)\right] \\
t=\frac{\left(\frac{C \nu}{\lambda}\right) 1 / \lambda \exp \left[-\frac{1}{\lambda}\right) D^{1 / \mu}}{A \sigma^{\alpha-\beta+1 / \mu c^{1+1 / \lambda}(1+1 / c \nu)}}\left[\exp \left(\frac{\mathrm{c} \nu}{\lambda}+\frac{1}{\lambda}\right)-\exp \left(\frac{c \nu_{0}}{\lambda}+\frac{\nu_{0}}{\nu \lambda}\right)\right]
\end{gathered}
$$

where $v_{0}$ is the initial value of $v$ which measures the initial value of hardness $\left(\log _{10} \sigma^{\star}\right), c=-(-\beta+1 / \mu)$ and $t$ is time.

The experimental creep data shown in Figure 8 are obtained under constant load. For constant load creep the stress increases with increasing strain. For creep calculation we take small strain intervals during which the stress is taken to be unchanged. At each interval with input of $\sigma$ and $\stackrel{*}{\sigma}$ the value of $v_{0}$ can be calculated (Equation 4). From Equation 7 for a given strain increment the increase in $\nu$ and therefore $\stackrel{*}{\sigma}$ can be calculated. With 
the value of $v$ and $v_{0}$ for this strain interval, the time interval can be evaluated from Equation 8 . For the next strain interval a new stress is calculated together with the $v$ value from the previous interval (corresponding to an increased hardness) we repeat the steps and obtain the strain vs time values of interest. The calculated creep curves (solid lines) are also given in Figure 8 .

In the creep calculation the $\log _{10} \sigma$ value used is the applied $\log _{10^{\circ}}$ plus $\Delta \log _{10^{\circ}}=0.1$ corresponding to the shift ${ }^{-}$ between the upper and lower curves in Figure 3 due to grain boundary sliding. For annealed specimen we assume that it is hardened instantaneousiy to a hardness value ( $\left.{ }^{*}\right)$ equal to that of the applied stress. We take therefore the initial * for the annealed specimen to be the value of applied stress plus a small number. For cold worked specimen knowing the plastic strain rate and the stress reached during cold working we can evaluate the value of $\stackrel{*}{\sigma}$ from Equation 2 . The value of $\log _{10^{\circ}}^{*}$ for the grain matrix of the 10 cold worked specimen corrected for the modulus effect to $250^{\circ} \mathrm{C}$ is 4.026 . For all the creep calculation the input required are the initial grain matrix stress and the initial grain matrix hardness. The values of the parameters in Equations 7 and 8 are given previously.

It is seen from Figure 8 that the calculated curves agree with measured data within the usual experimental scatter in creep experiments. The creep specimen used has a small diameter of 0.1 inch and a gage length of 0.75 inch. We suspect that the effects of the non-uniformity in microstructure and in the specimen diameter along the gage length may cause most of the scatter 
in creep data. The effects of non-uniform diameter seem to be more pronounced in cold-worked specimen due to plastic instability. Physically the present creep analysis is based on (1) the work hardening properties and the strain rate sensitivity of a material depends only on current values of stress and hardness $\left(\log _{10^{\circ}}\right.$ and $\log _{10^{\circ}} \stackrel{\star}{)}$ or stress and plastic strain rate and (2) during creep deformation under the experimental conditions of the present work, there is no recovery of deformed structure and the structure change results only from work hardening due to strain increments. Mathematically the $\varepsilon_{p}$ vs $t$ values obtained from Equations 7 and 8 in the $\log _{10} \epsilon_{p}$ vs $\log _{10}$ t plot will have a temperature independent shape for given $\log _{10^{\sigma}} \sigma \mathrm{G}$ and initial $\log _{10^{\circ}}{ }^{*} \mathrm{G}$. Experimentally the results given in references 22 and 23 on aluminum alloys show this behavior that in a $\log _{10}$ vs $\log _{10}\left(\mathrm{t} \mathrm{e}^{-Q / R T}\right)$ plot for a given stress and initial structure the creep data from different temperatures overlap. The temperature compensating behavior shown in references 22 and 23 suggests alsouthelabsence of recovery during creep deformation. If both work hardening and recovery are operating, it is difficult to conceive that they will have same temperature dependence to produce the observed results.

The absence of recovery during creep deformation suggests that the shape of $\epsilon_{p}$ vs $t$ creep curve will depend strongly on the work hardening properties of a material in the experimental conditions of the present work. For a cold worked material the * value is high. At a low applied stress according to Equation 5 the value of the parameter $\Gamma$ will be very small. In this case we expect little work hardening during creep deformation and 
Ep vs t data will be close to a straight line (curves 2 and 3 , Fig. 8). For an annealed material the initial $\stackrel{\star}{a}$ value will be low resulting in a large $\Gamma$ value. During creep deformation work hardening will be important initially and decreases with increasing strain or hardness. In the latter case the creep curve will first show a region with decreasing strain rate and gradually approaches a nearly constant strain rate (curve 1, Eig. 8).

It should be noted that at the initial stage of creep deformation, transient effects $(3,7)$ wi11 be present. The transient effects are not included in the present creep analysis. The present analysis will produce a primary stage due to the work hardening effects as discussed above. For cold worked specimens the work hardening effects are small. The transient effects can be identified by noting that the initial portion of the creep data exhibit higher strain rates compared to those of the calculated curves in Fig. 8 .

The activation energy determined from creep data obtained using the same applied stress and initial structure ${ }^{(22,23)}$ according to the discussion given previously will be the same as that determined in the present work. The low value activation energy measured at lower temperatures may correspond to that for dislocation pipe diffusion ${ }^{(24)}$. As temperature increases, the measured value may reflect the importance of bulk processes (15).

In summary the present work has found:

1) IID0 aluminum alloy exhibits the behavior of mechanical equation of state in monotoric loading in a temperature range up to above one half of its absolute melting temperature. 
2) The activation energy for plastic deformation in this temperature range is found to depend only on temperature and is independent of stress and plastic strain rate.

3) The contribution of grain boundary sliding in plastic deformation is identified.

4) The activation energy for grain boundary sliding is found to be close to that for grain matrix deformation. The stress dependence of grain boundary sliding rate exhibits norNewtonian behavior.

5) In the temperature range of the present work the recovery of deformed structure due to grain boundary migration is found to be absent.

6) Using the measured work hardening and strain rate sensitivity properties, the calculated creep behavior shows similar features as those observed experimentaliy.

\section{ACKNOWLEDGEMENT}

This work is supported by the U. S. Atomic Energy Conmission and by the Materials Science Center at Cornell University sponsored by the National Science Foundation. The authors wish to acknowledge their'discussion with E. W. Hart and the assistance of B. Addis. F. H. Huang and R. S. Chao participate in creep calculations and measurements. 


\section{APPENDIX}

\section{Creep Ana1ysis}

The following creep calculations are restricted to the conditions where grain boundary is completely relaxed and the deformation behavior of 1100 aluminum alloy can be represented by the parallel model proposed by Hart ${ }^{(16)}$. Under these conditions the applied stress and plastic strain rate for a particular hard ness will fall on the portion of the curves equivalent to the lower curve in Figure 3 where it overlaps the composite curve. The grain matrix properties of the material are represented by the upper solid curve in Figure 3 .

We begin the calculations with Equations $1,2,4,5$ and 6 and the associated constants in the text. The work hardening parameter and the strain rate parameter are given by Equations 4 and 5 in terms of grain matrix $\sigma$ and ${ }_{\sigma}^{*}$. We integrate Equation 1 to obtain plastic strain vs tine relationship for constant stress. From Equations 1 and 5 at constant stress we write

$$
\Gamma \mathrm{d} \varepsilon_{p} l_{\sigma}=-\left.\frac{\mu v}{\mu-v} \mathrm{~d} \ln \dot{\varepsilon}_{p}\right|_{\sigma}
$$

Differentiating Equations 2 and 4 at constant stress and using Equation 4,

$$
\text { d }\left.\ln \dot{\varepsilon}_{p}\right|_{\sigma}=\frac{-(\mu-v)}{\lambda \mu v} d v
$$

Combining Equations $A 1$ and $A 2$ and using Equation 5 we obtann, 


$$
\left.d \varepsilon_{p}\right|_{\sigma}=\frac{d v}{\lambda \Gamma}=\frac{d v}{\lambda A \sigma^{\alpha} / \sigma^{*}}
$$

Integrating $A 3$ gives the relationship between $E$ and $v$

$$
E_{p}=\frac{1}{A B \sigma^{\alpha-\beta}}\left[\exp \left(\frac{\beta \nu}{\lambda}\right)-\exp \left(\frac{B \nu_{o}}{\lambda}\right)\right]
$$

where $v_{0}$ is given by Equation 4 in terms of initial grain matrix $\stackrel{*}{\sigma}$ and grain matrix stress. From Equations 2,4 and 6 the plastic strain rate is

$$
\dot{\varepsilon}_{p}=\frac{\sigma^{1 / \mu}}{D^{1 / \mu}} \exp \left(\frac{u}{\lambda \mu}\right)\left(\frac{v}{\lambda}\right)^{-1 / \lambda}
$$

Combining Equations $\mathrm{A} 3$ and $\mathrm{A} 5$ the retationship between time $t$ and $v$ is given,

$$
\begin{aligned}
& d t=\left.\frac{1}{\varepsilon_{p}} \mathrm{~d} \varepsilon_{p}\right|_{\sigma} \\
& =\frac{D^{1 / \nu_{(}}\left(\frac{v}{\lambda}\right)^{1 / \lambda} d v}{\lambda A \sigma^{\alpha-\beta+1 / \mu} \exp \left[\frac{v}{\lambda}\left(-\beta+\frac{1}{\nu}\right)\right]}
\end{aligned}
$$

Let $X=\frac{c \nu}{\lambda}$ and $c=-\left(-\beta+\frac{1}{\psi}\right)$ and integrate

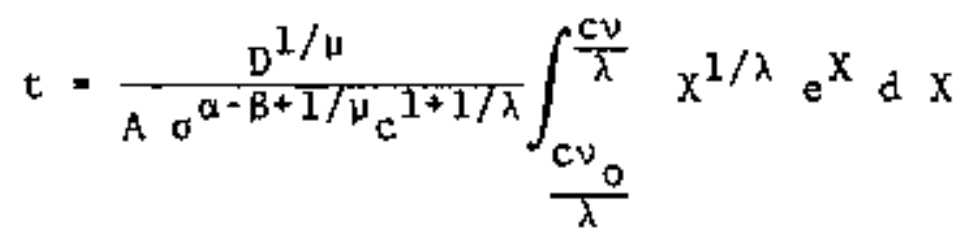

We expand the logarithm of the integrand and around the upper limit and take only the first term, Equation A7 becomes 


$$
\begin{gathered}
t=\frac{D^{1 / \mu}\left(\frac{C v}{\lambda}\right)^{1 / \lambda} e^{-1 / \lambda}}{A \sigma^{\alpha-\beta+1 / \mu} c^{1+1 / \lambda}} \int_{\frac{C v_{0}}{\lambda}}^{\frac{C v}{\lambda}} \exp \left[x\left(1+\frac{1}{C \nu}\right)\right] d x \\
=\frac{D^{1 / \mu}\left(\frac{C \nu}{\lambda}\right)^{1 / \lambda} e^{-1 / \lambda}}{A \sigma^{\alpha-\beta+1 / \mu} c^{1+1 / \lambda}\left(1+\frac{1}{c \nu}\right)}\left[\exp \left(\frac{C \nu}{\lambda}+\frac{1}{\lambda}\right)-\exp \left(\frac{c v_{0}}{\lambda}+\frac{\nu_{0}}{\mu \lambda}\right)\right]
\end{gathered}
$$

Given initial $v_{0}$ we can calculate the value of $v$ from Equation A4 at desired $E_{p}$. From the calculated $v$ and Equation $A 8$ the time required can be evaluated. The approximation used in the integration of Equation $A 7$ introduces less than $10 \%$ error.

The calculations given here apply also the the case where the deformation is controlled by grain matrix processes (upper solid curve in Fig. 3). In the region where both grain matrix processes and grain boundary processes are important the present creep calculations have to be modified. 


\section{REFERENCES}

1. E. W. Hart, Acta Met., 18, 599 (1970).

2. E. H. Hart, "On the Existence of a State Property in the Plastic Deformation of Metals", General Electric Report No. 69-C-362 (Oct. 1969).

3. H, D. Solomon and E. W. Hart, Acta Met., 21, 195 (1973).

4. H. Yamada and Che-Yu Li, "Stress Relaxation and Mechanical Equation of State in Nickel and TD Nickel". Presented at J. E. Dorn Memorial Symposium on "Rate Processes in plastic Deformation", Fall Meeting, ASM, 1972, to be published in proceedings of symposium.

S. H. Yamada and Che-Yu Li, Acta Met., 22, 249 (1974).

6. H. Yamada and Che-Yu Li, Met. Trans., 44, 2133 (1973).

7. 6. Wire, H. Yanada and Che-Yu Li, Acta Met., 22, 505 (1974).

8. G. L. Wire, F. V. Ellis and Che-Yu Li, "Work Hardening and Mechanical Equation of State in Some Metals in Monotonjc Loading", AEC Report \#COO-2172-4, Cornell University (1973), submitted to Acta. Met.

9. I. S. Servi and N. J. Grant, J. Metals, 191, 909 (1951).

10. D. Lee and E. W. Hart, Met. Trans., 2, 1245 (1971).

11. E. W. Hart, Private communication.

12. E. W. Hart, "The plastic Equation of State at HIgh and Low Temperatures", presented at $J$. E. Dorn Memorial Symposium on "Rate Processes in Plastic Deformation" Fall Meeting, ASM, 1972, to be published in the proceedings of the symposium.

13. P. M. Sutton, Phys. Rev., 91, 816 (1953).

14. E. Aerts, P. Delavignetti, R. Sicms and S. Amelinckx, J. Appl. Phys., 33, $3070(1962)$.

15. J. E. Dorn and N. Jaffe, Trans AIME, 221, 229 (1961).

16. E. W. Hart, Acta Met., 15, 1545 (1967).

17. E. W. Hart, "Intergranular Failure", General Electric Report No. $69-\mathrm{C}-334$ (Sept. 1969). 
18. C. A. P. Horton, Acta Met, , 18, 1159 (1970).

19. C. A. P. Horton, Acta Met., 20, 477 (1972).

20. F. B. Cuff, Jr. and N. J. Grant, Trans AIME, 212, 355 (1958).

21. T. V. Cherian, P. Pietrokowsky and J. E. Dorn, Metals Transactions AIME, $\underline{185}, 948$ (1949).

22. L. Raymond and J. E. Dorn, Tran AIME, 230, 560 (1964) .

23. R. E. Frenke1, D. D. Sherby and J. E. Dorn, Trans. ASM, $\underline{47}, 632(1955)$.

24. T. E. Volin, K. H, Lie and R. W. Balluffi, Acta Met., $19,263^{\circ}(1971)$. 


\section{FIGURE CAPTIONS}

Figure la

Figure Ib

Figure Ic

Figure 2

Figure 3

Figure 4 $\log _{10}$ (stress) vs $\log _{10}$ (strain rate) data for 1100 aluminum alloy at roon temperature and several plastic strain levels. $\log _{10}$ (stress) vs $\log _{10}$ (strain rate) data for 1100 aluminum alloy at $150^{\circ} \mathrm{C}$ and several plastic strain levels.

$\log _{10}$ (stress) vs $\log _{10}$ (strain rate) data for 1100 aluminum alloy at $200^{\circ} \mathrm{C}$ and several plastic strain levels.

Constant hardness log $_{10}$ (stress) vs $\log _{10}$ (strain rate) data for 1100 aluminum alloy at several temperatures. Stress values are modulus corrected for temperature. Stress scale shown is that for room temperature data.

Composite constant structure $\log _{10}$ (stress) vs $\log _{10}$ (strain rate) data for 1100 aluminum alloy obtained by translation of constant hardness $\log _{10^{\circ}}-\log _{10} \dot{\varepsilon}_{p}$ curves of Figure 2 along $\log _{10} \dot{\varepsilon}_{p}$ axis to match overlapping segments. $\log _{10}$ (stress) and $\log _{10}$ (strain rate) scales are for room temperature data.

Calculated $\log _{10}$ (grain boundary stress) vs $\log _{10}$ (grain boundary strain rate) data for 1100 aluminum alloy at several temperatures based on a parallel model for grain boundary sliding and data shown in Figure 2. Stress values are modulus corrected for 
Figure. 4

(cont inued)

temperature. Stress scale shown is that for room temperature data.

Figure 5

Composite grain boundary sliding curve of $\log _{10}$ (grain boundary stress) vs $\log _{10}$ (grain boundary strain rate) data for 1100 aluminum alloy. $\log _{10}$ $\left(\sigma_{S}\right)$ and $\log _{10}\left(\dot{\mathrm{e}}_{\mathrm{g}}\right)$ curves of Figure 4 are translated along the $\log \dot{\varepsilon}_{g}$ axis with the amount of strain rate shift determined by the activation energy for grain matrix deformation. $\log _{10} \sigma_{S}$ and $\log _{10} \dot{\varepsilon}_{g}$ scales are for room temperature.

Figure 6 Constant $r$ or work hardening parameter points correlated with $\log _{10^{\circ}}$ and $\log _{10^{\sigma} / \mathrm{o}}$ for 1100 aluminum alloy where ${ }^{*}$ is the hardness parameter.

Figure 7 Graphical representation of work hardening and strain rate sensitivity parameter for grain matrix behavior of 1100 aluminum alloy based on Equation 2 and 5 covering a wide range of temperature, strain rate and deformed structure. The $\log _{10}$ $\left(\Gamma \stackrel{*}{\sigma}^{B} / A\right)^{1 / \alpha}$ scale shown provides a convenient method of evaluating the work hardening parameter. Figure 8 Calculated constant load creep curve for annealed and cold worked I100 aluminum alloys at given conditions to compare with measured creep data. $\sigma_{i}$ is initial applied stress. 


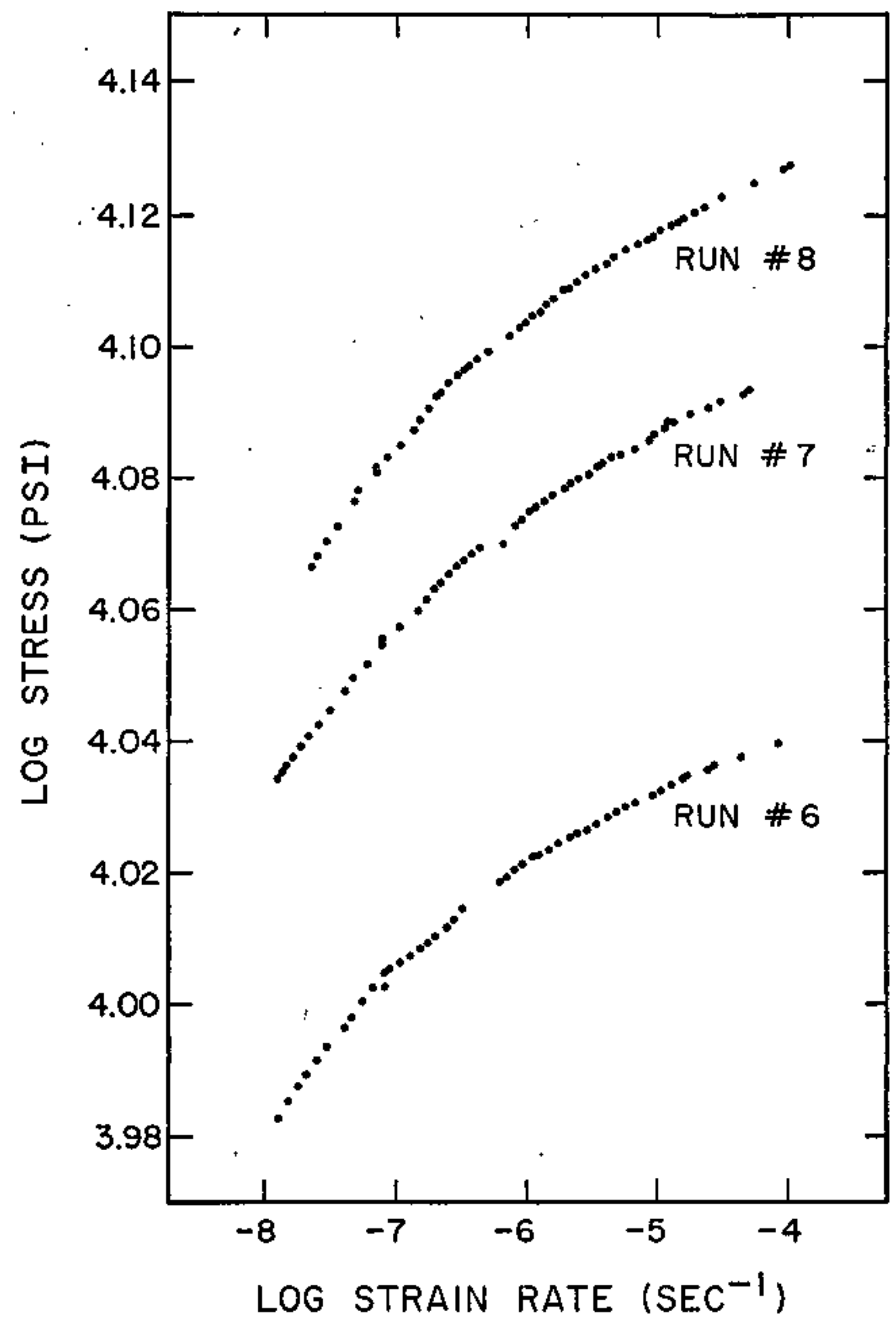

Figure 1a 


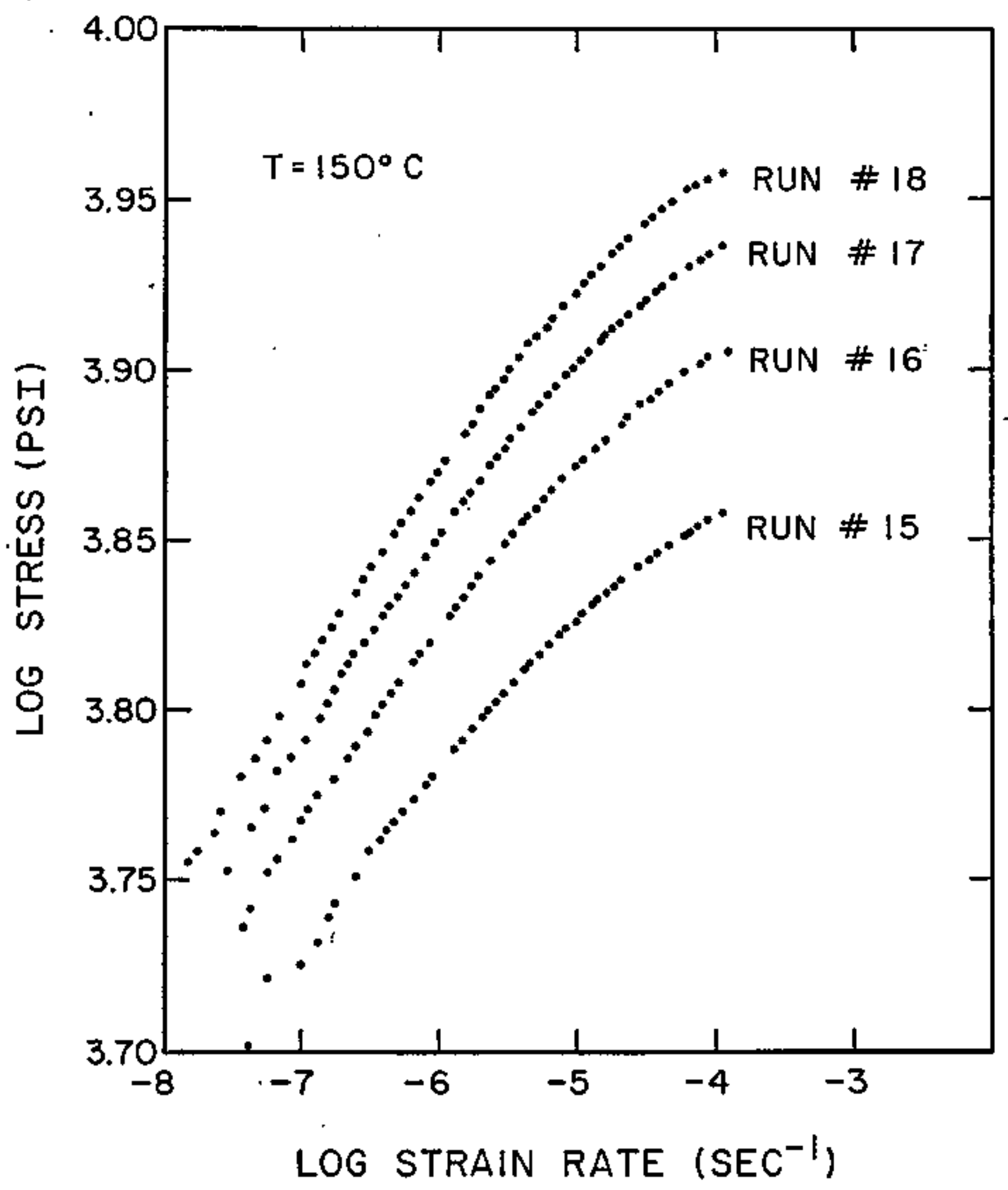

Figure $1 b$ 


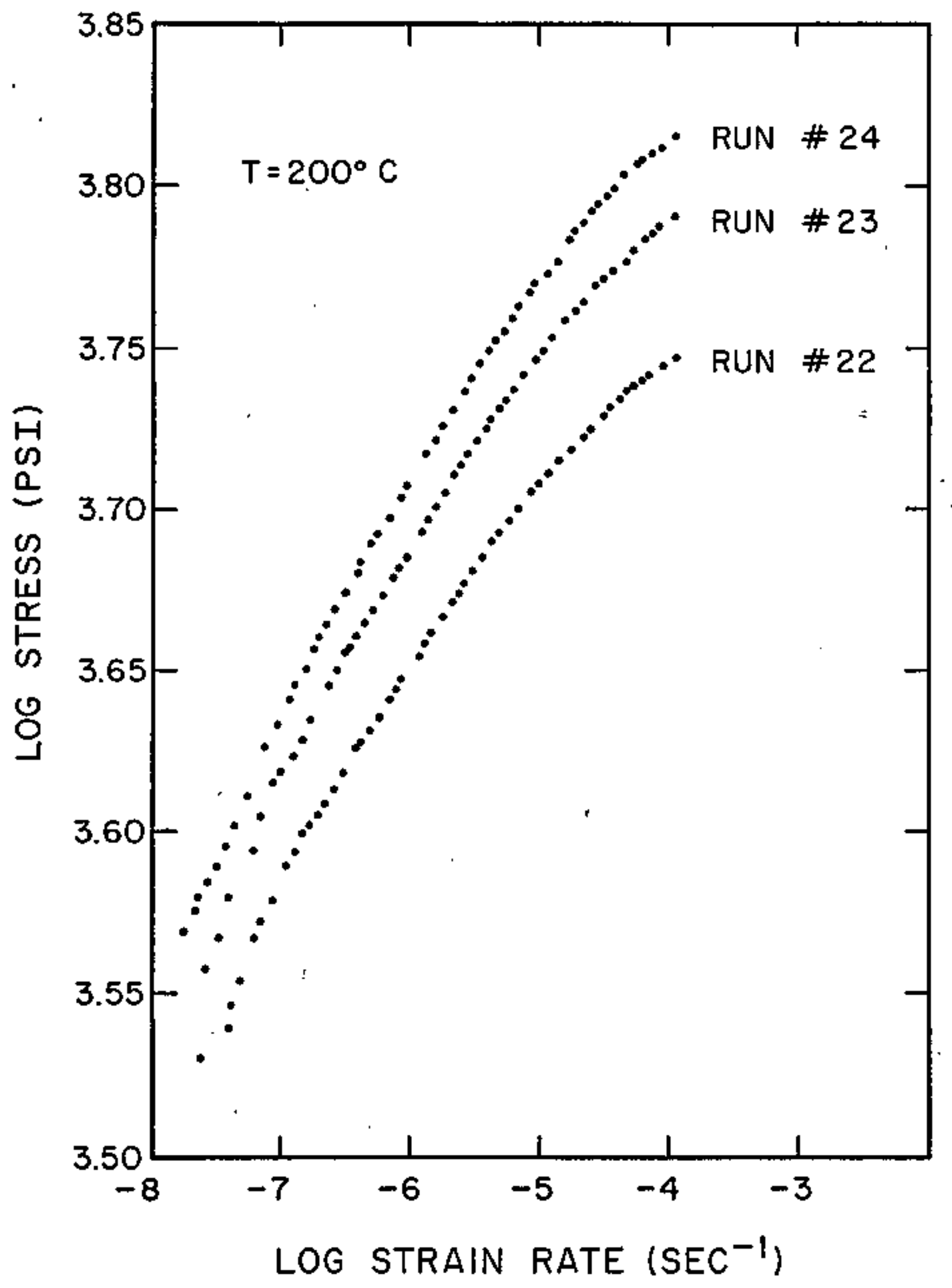

Figure lc 


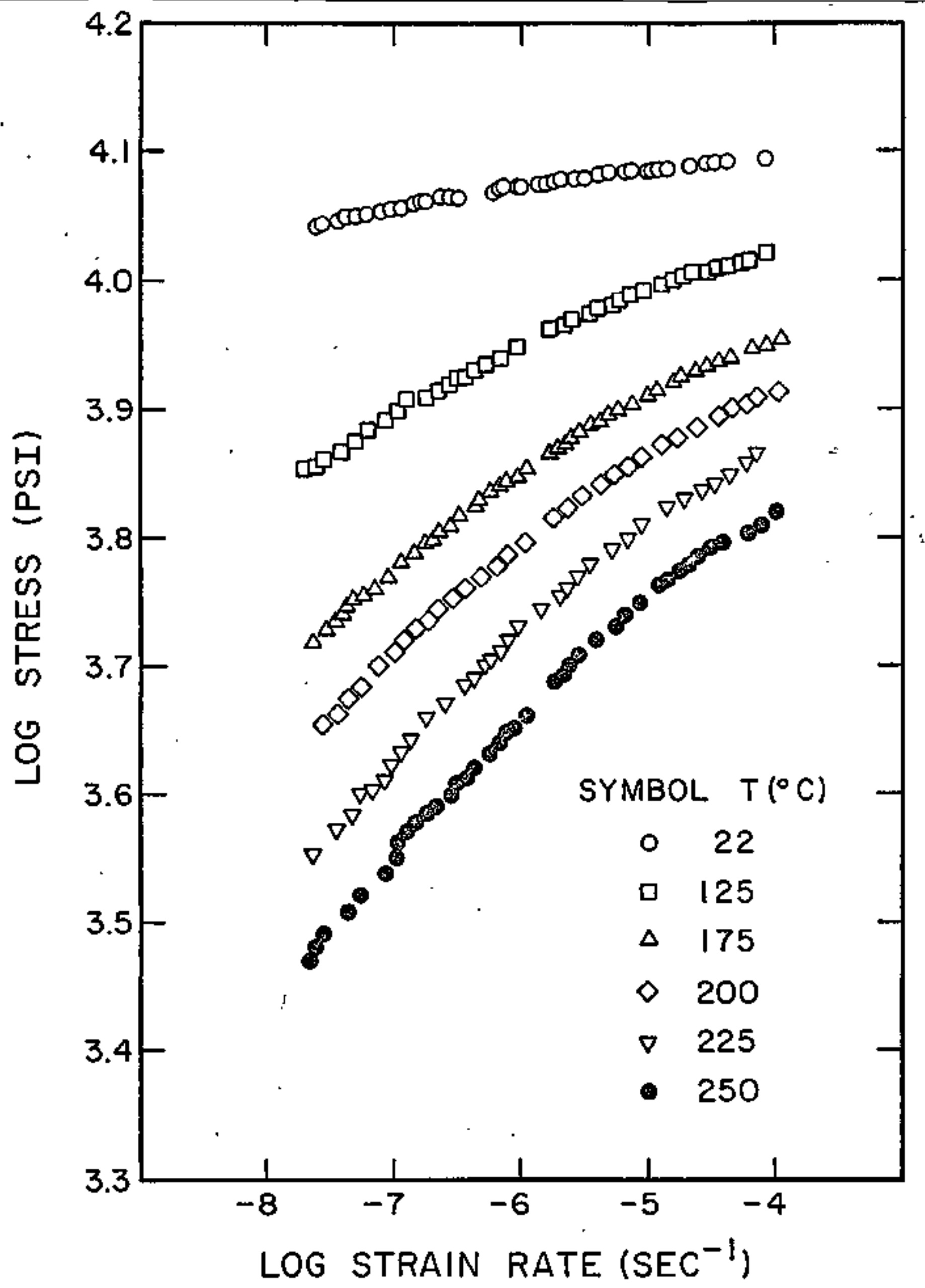

Figure 2 


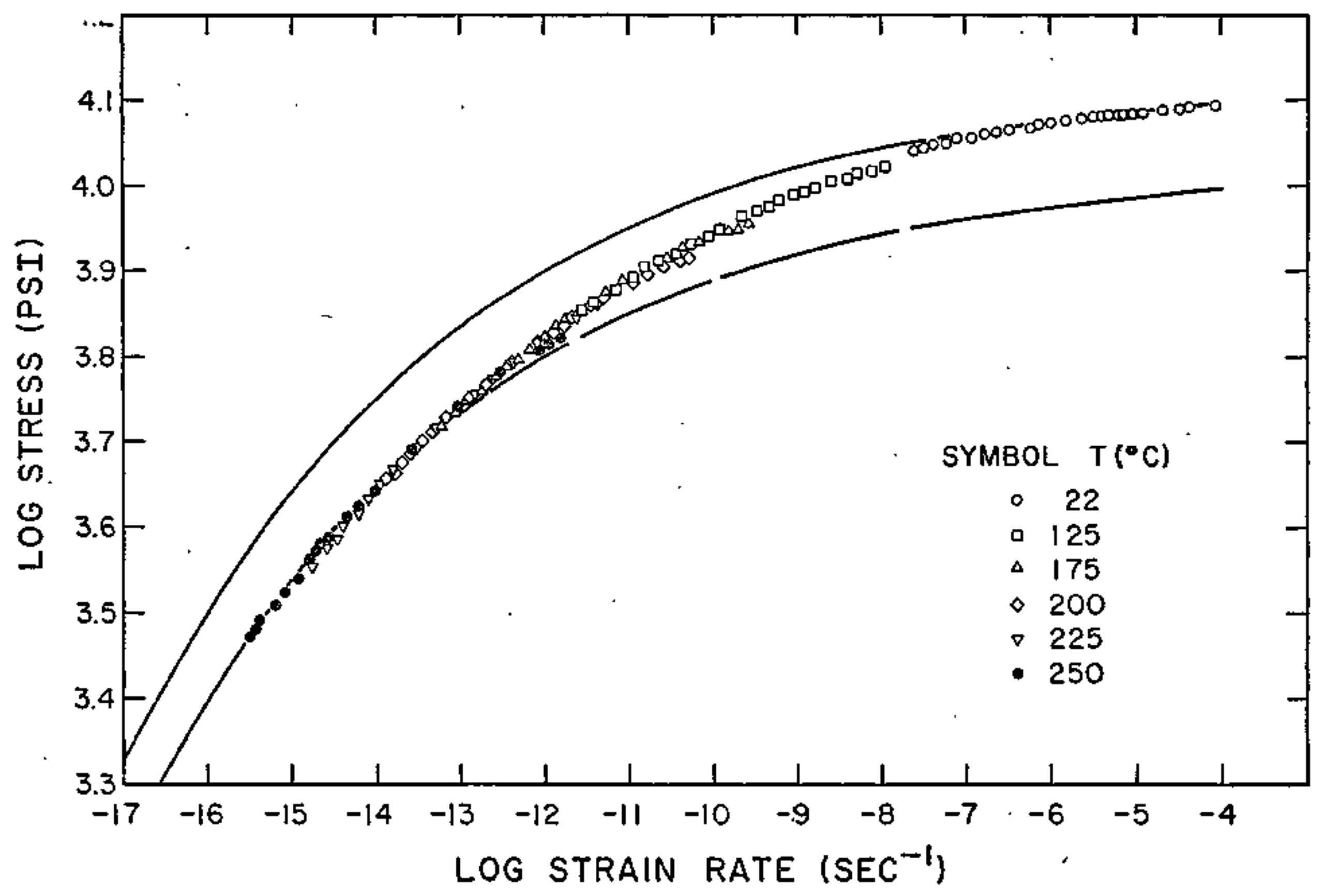

Figure 3 


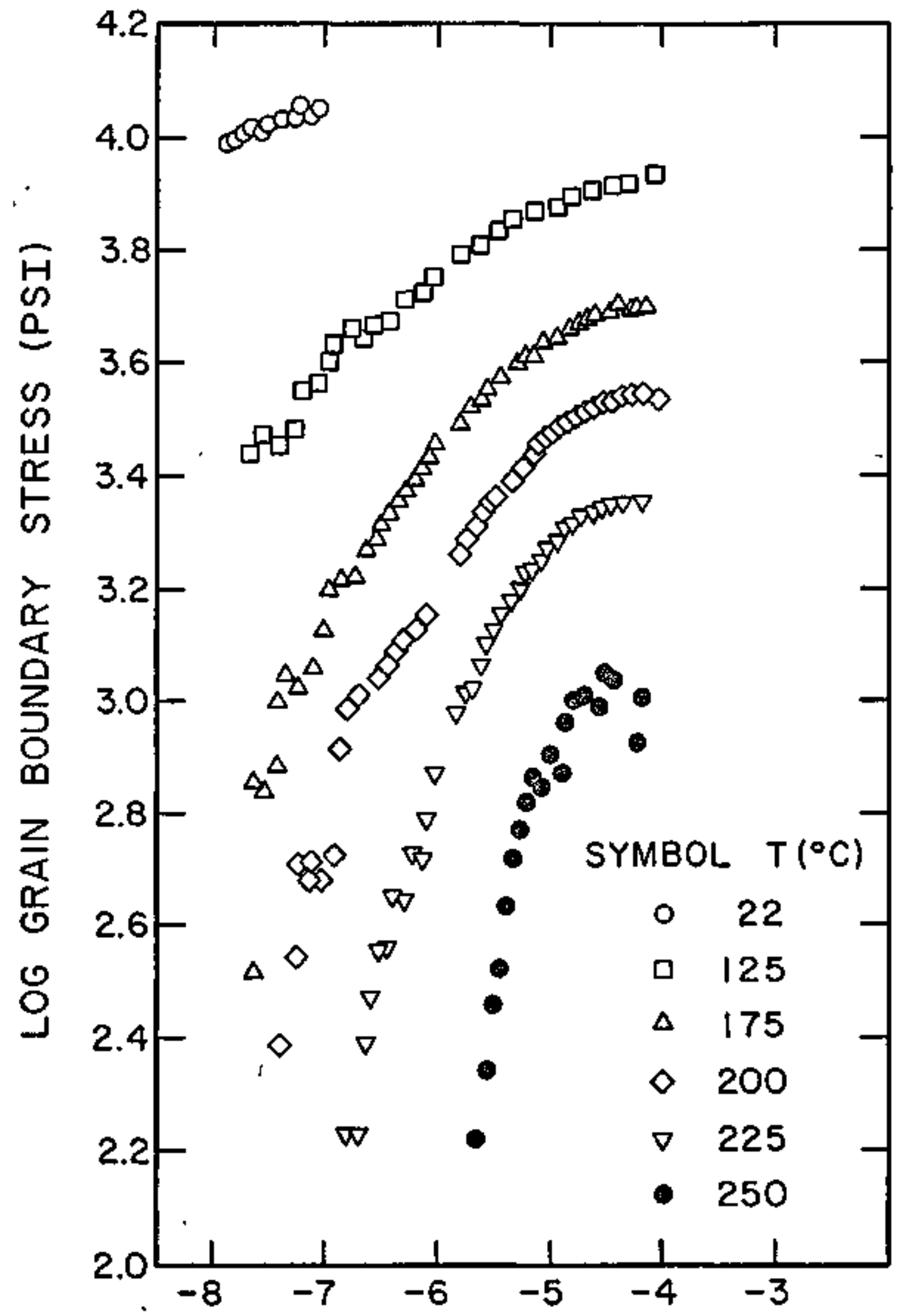

LOG GRAIN BOUNDARY STRAIN RATE (SEC ${ }^{-1}$ ) 


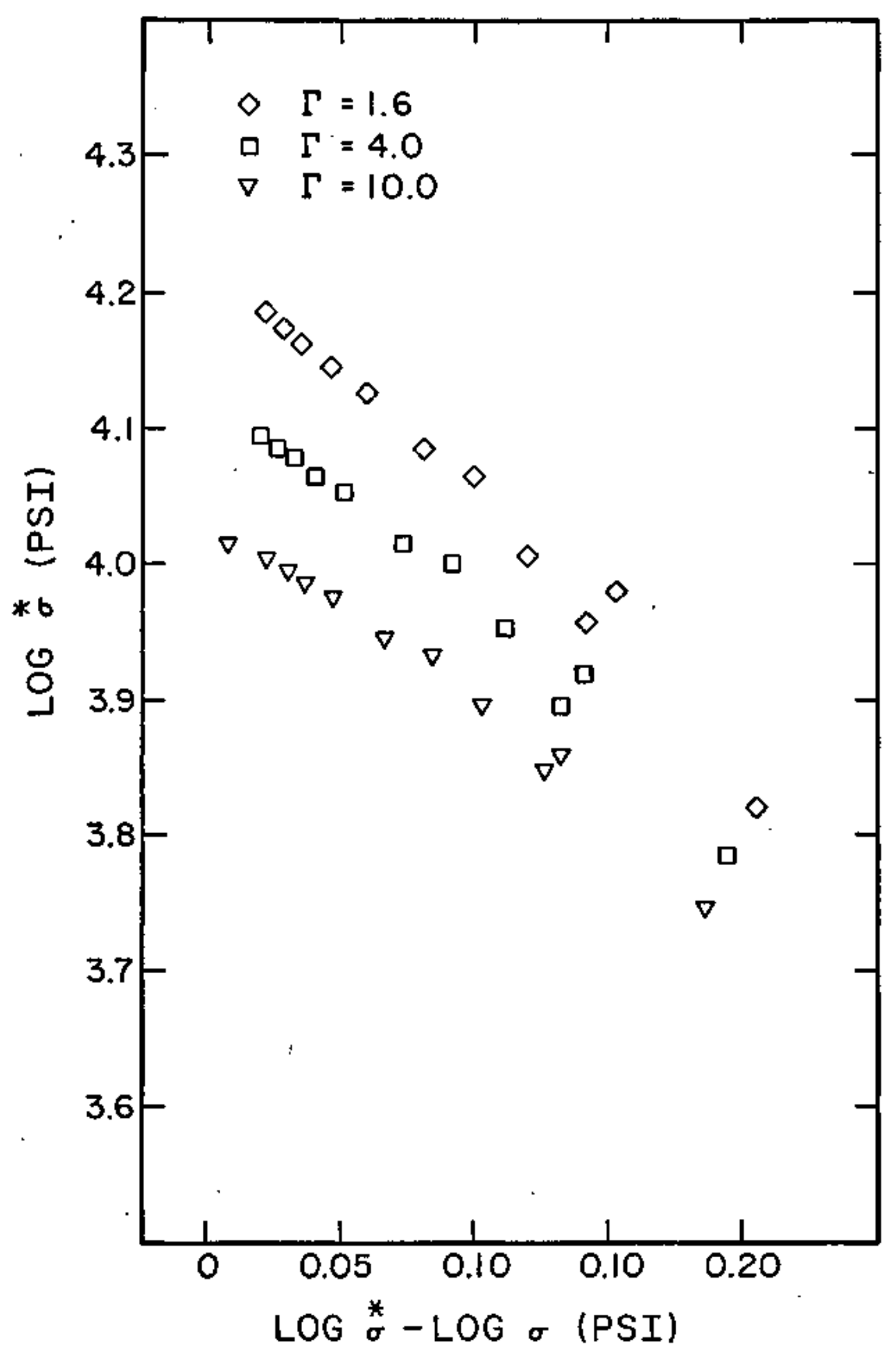

Figure 6 


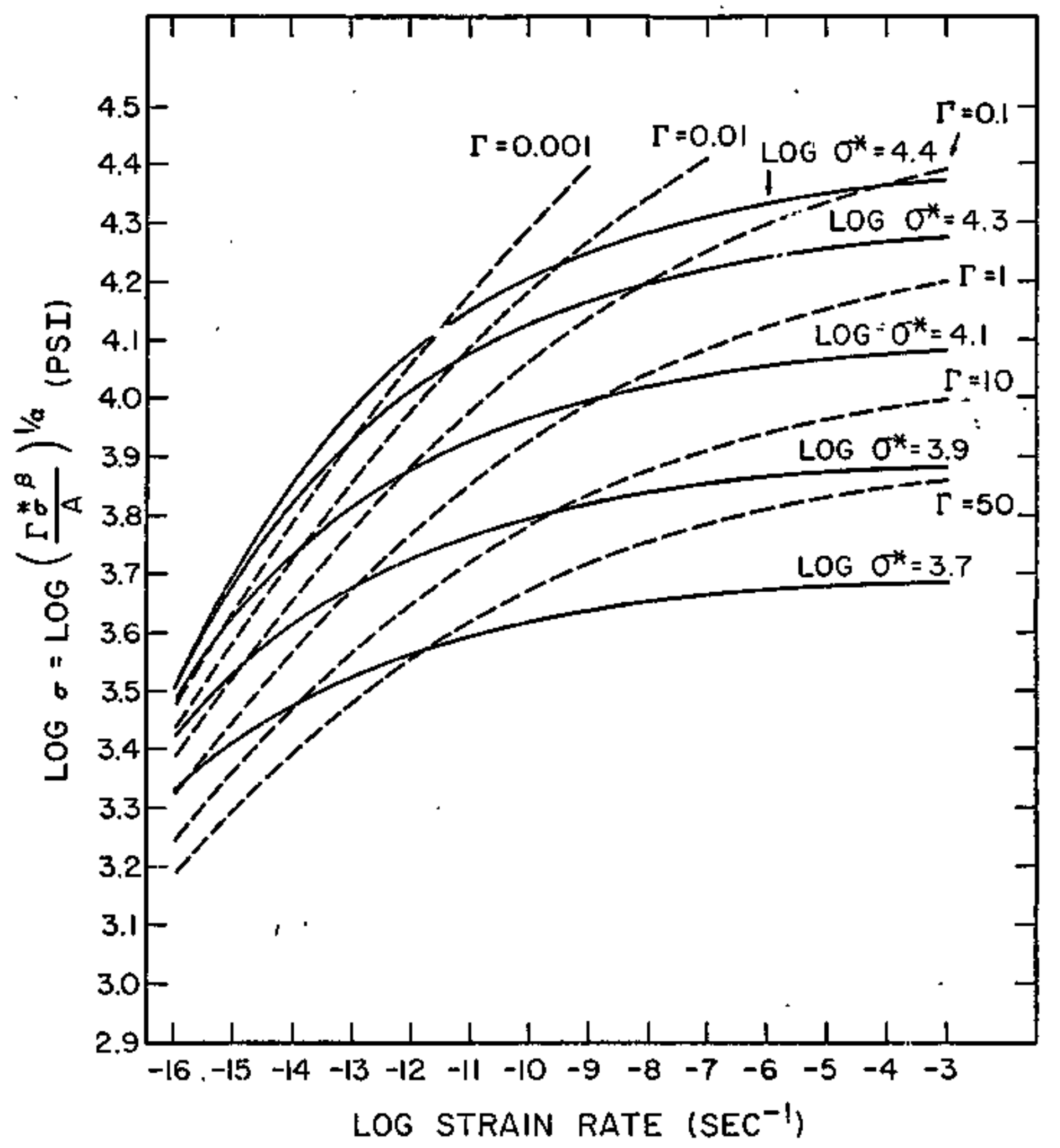

Figure 7 


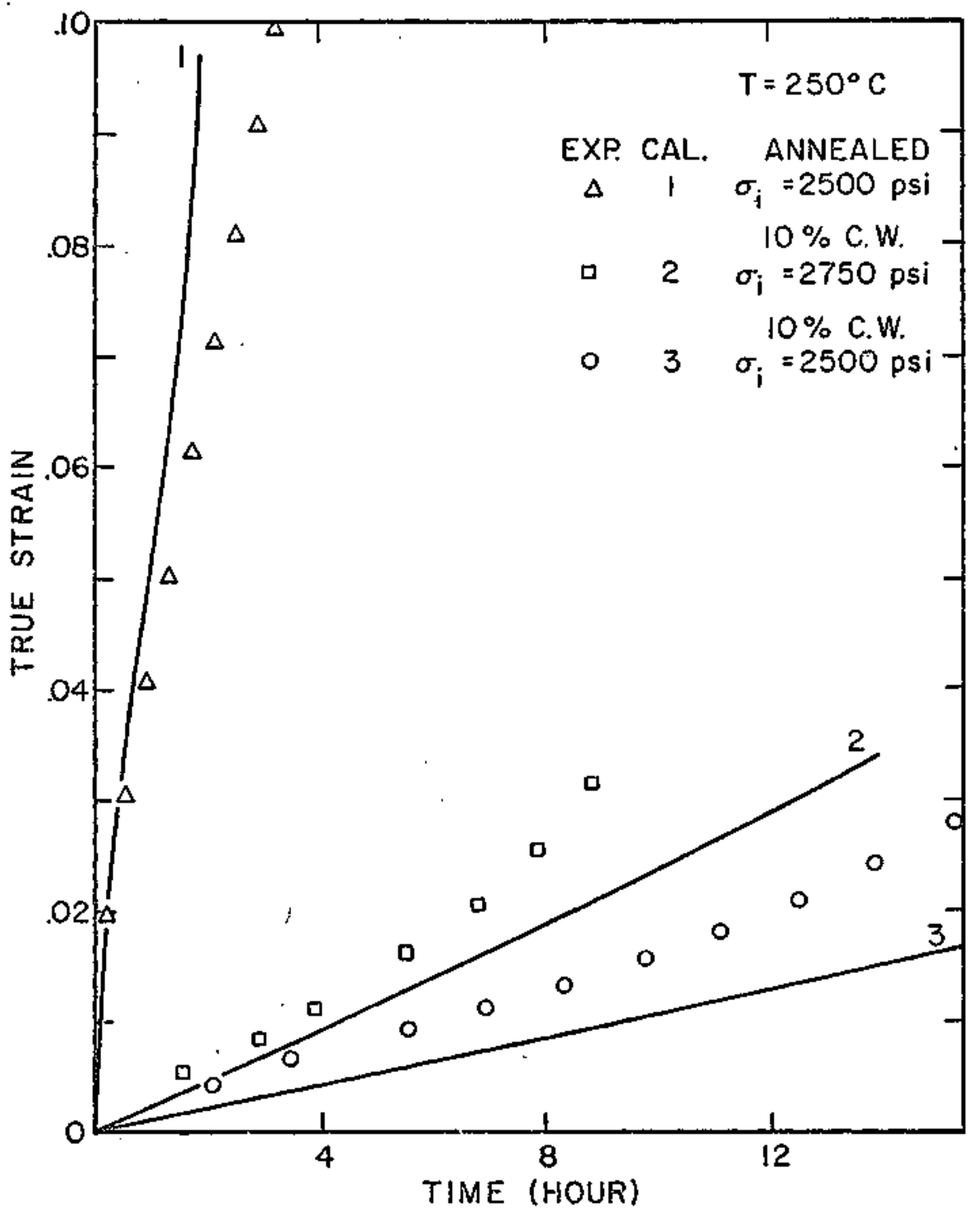

Figure 8 